\title{
Massive Machine Type Communication and Satellite Integration for Remote Areas
}

\author{
Muhammad Asad Ullah, Konstantin Mikhaylov, and Hirley Alves \\ Centre for Wireless Communications, University of Oulu, Finland \\ firstname.lastname@oulu.fi
}

\begin{abstract}
Despite immense progress along the different tracks, the wireless connectivity for machine-applications in remote areas is still very challenging. To address this vital need, in this paper, we discuss and advocate the convergence between the low power wide area network (LPWAN) grade massive machine-type communication (mMTC) wireless technologies and the satellite (especially low earth orbit, LEO) systems. In the paper, we discuss the alternative implementation approaches allowing for such convergence and highlight some of their pros, cons, and challenges. Furthermore, we obtain a more in-depth insight into the matter by simulating and analyzing the LoRaWAN LPWAN sensors' performance served by an LEO satellite-based gateway. Our results demonstrate the feasibility of such a system and illustrate some of the relevant trade-offs between the network configurations and communication performance. These results motivate to take a more profound look at such systems and the challenges they introduce. We highlight some of them and the potential directions for further studies in the final sections.
\end{abstract}

\section{INTRODUCTION AND VISION}

The past years have delivered immense technology progress in the Internet-of-Things (IoT) related fields, with connectivity being among the most illustrative examples. The use cases, which are jointly referred to as massive machine-type communications (mMTC) and demand cost-effective wireless connectivity for a large number of devices with limited connectivity requirements, have been addressed through the introduction of the several low-power wide-area network (LPWAN) technologies both within the $3 \mathrm{GPP}$ and beyond it [1]. In 3GPP, the cellular IoT has become one of the notable offerings of fifthgeneration $(5 \mathrm{G})$ cellular technologies and is currently being deployed worldwide. However, despite all this progress, the MTC connectivity today is still not ubiquitous. Specifically, being already widespread in urban and suburban regions, the remote areas are still poorly covered by MTC technologies. Below, we offer just three motivation examples:

- According to the World Bank, around 476 million indigenous people live in more than 90 countries. Indigenous societies are at higher risk due to the impact of environmental change, disaster floods, and epidemic such as e.g. coronavirus. The availability of MTC in indigenous regions can enable novel applications for nature monitoring, improving safety and quality of living, and boosting productivity.

- The innovative autonomous smart agriculture, husbandry and irrigation solutions based on the accurate environment, soil, and dynamic weather conditions tracking will maximize the product yield while optimizing the use of human labour and minimizing overhead expenses. This may also enable farming in the regions where traditional agriculture has been inefficient - with the Sahara desert being just one example.

- Marine transportation is today accounting for more than $90 \%$ of the global trade, and these days there is a strong trend for advancing the vessel's autonomy. Also, according to the Allianz Global Corporate \& Specialty data, despite the decrease of the total number of vessels lost in the past years, the number of casualties and technical incidents increases. The IoT solutions for monitoring ship structure integrity and tensions may help to fight these.

Due to reasons of technical, economic and legislative nature, the conventional terrestrial MTC networks cannot cover the entire globe. Unfortunately, this situation will not likely change in the near future. Therefore, in this article, we investigate the feasibility and potential performance of enabling MTC in remote locations over Non-Terrestrial Networks (NTNs), and, specifically, the satellite networks (Fig. 1). Accordingly, the contribution of our work is threefold. First, we offer an investigation of the pros and cons behind MTC over satellite networks, together with its alternative architectures. Second, we suggest integrating low earth orbit (LEO) satellites with the LoRaWAN LPWAN technology while discussing the relevant changes for the protocol and how these can be addressed. Third, we develop a simulation model and obtain insight into the satellite-based LoRaWAN networks' performance limits and the respective trade-offs. The presented results illustrate that satellite-based MTC is feasible, highlight some relevant trade-offs, and motivate further studies in this field.

The rest of this paper is organized as follows. Section II provides an insight into the background of massive MTC (mMTC) and satellite technologies. In Section III we discuss the two alternative implementation approaches, including their pros and cons. In Section IV, we analyze and present the illustrative numerical results for the selected scenario, implying direct data transfer from ground sensors to a satellite gateway. Finally, in Sections V and VI we point out open questions and further research directions and summarize our key results.

\section{TECHNICAL BACKGROUND AND RECENT DEVELOPMENTS}

In the two following subsections, we briefly discuss the technical background, focusing on the recent developments for mMTC and LPWAN, and satellite technologies. 


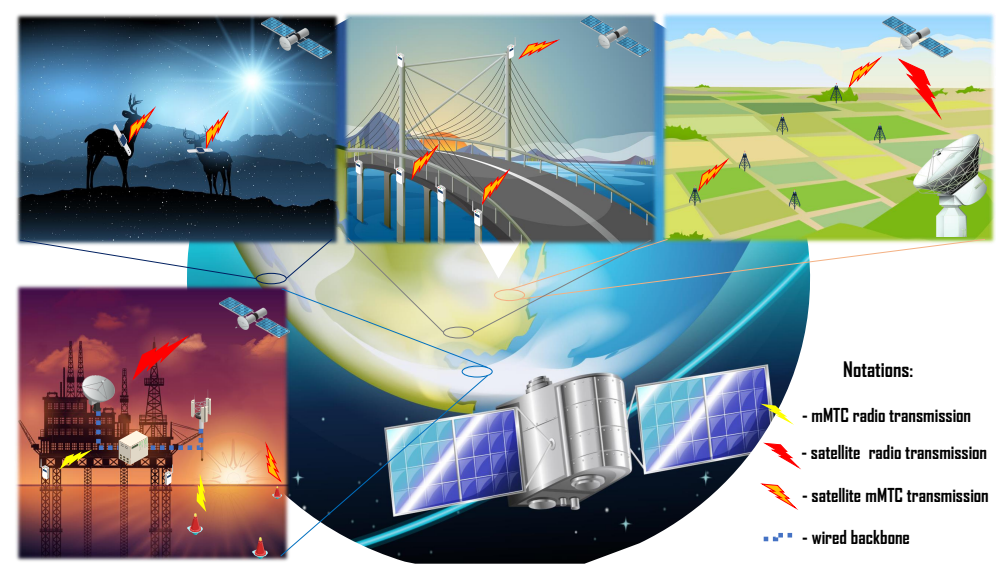

Fig. 1. Our vision: ubiquitous mMTC-satellite powered remote areas and application examples.

\section{A. mMTC technologies}

The introduction of the LPWAN technologies and their massive deployment in the past seven years have drastically changed the landscape of mMTC connectivity, delivering a paradigm shift from ad-hoc and mesh solutions (based, e.g., on ZigBee or WiFi) towards the cellular-like architectures. As of today, the three LPWAN technologies dominate the market, namely Sigfox, LoRaWAN and Cellular-IoT.

Both Sigfox and LoRaWAN represent clean-state connectivity technologies operating within the sub-GHz license-free frequency bands. Both technologies imply star-of-stars-based network topology and, primarily, Aloha-like media access. To support the long-range connectivity and scalability, Sigfox bases on ultra-narrowband (having bandwidth as low as $100 \mathrm{~Hz}$ in the uplink) radio signals, randomly selecting the carrier frequency and limiting the maximum payload to only 12 bytes [2]. The LoRaWAN technology uses the proprietary chirp spread spectrum based modulation-coding scheme named LoRa. The spreading factor (SF) of the signal can be varied, thus trading the on-air-time for the maximum connectivity range. The LoRa signals modulated with different SFs are quasi-orthogonal, which, along with a random selection of a frequency channel for uplink transmission, facilitates the scalability [3]. Notably, neither Sigfox nor LoRaWAN (i) implies an association of an MTC device to a specific gateway, thus avoiding handovers and related signalling overhead costs, and (ii) supports internet protocol (IP) over the on-air links.

In contrast to LoRaWAN and Sigfox, the Cellular-IoT technologies - the umbrella-term consolidating IoT and M2Mfocused radio access technologies developed within the 3GPP are intended to operate in the licensed frequency bands. Starting with the introduction of categories 1 and 0 (Cat. 1 and Cat. 0, Rel. 8 and 12, respectively), the LTE-M (Cat, M1/M2), NB-IoT (Cat NB1/NB2), and EC-GSM followed in Rel. 13 and 14. While NB-IoT and LTE-M present a deep evolution and adaptation of the LTE technology to address the common needs of mMTC and have been already commercially deployed in many regions of the world, ECGSM bases on the GSM technology and got a rather limited commercial use for now. Basing primarily on the orthogonal frequency-division multiplexing for downlink and frequency division multiple access for uplink communications, NB-IoT employs significantly simplified LTE-based procedures for accessing the radio channel and transferring the data. The repetition of the symbols is actively used to increase possible communication distances. Further enchantments for the energy consumption (e.g., PSM, RAI) and data transfer robustness and performance increase (e.g., TBS and HARQ, EDT) have been introduced in the subsequent specification releases. One of the differences between Sigfox/LoRaWAN and NB-IoT/LTE-M is the support of the IP-based connectivity by the latter.

\section{B. Satellite technologies}

The satellite systems are typically classified in the three major groups based on the orbit altitude above the Earth surface, namely Low Earth Orbit (LEO, altitude below $2000 \mathrm{~km}$ ), Medium Earth Orbit (MEO, 2000-10000 km), and Geostationary Orbit (GEO, $35000 \mathrm{~km})$. As a rule of thumb, the elevation of the orbit's altitude increases attenuation and the time for a radio signal reaching a satellite. Still, it raises the coverage of a single satellite and its orbital lifetime. To give an example, while at least 15 LEO satellites are required for global coverage, three GEO satellite can do the job.

According to the report of the Union of Concerned Scientists, there are 3372 active satellites on all orbits as of January 1, 2021. The earth orbit is dominated by 2612 LEO satellites (increasing by $77.92 \%$ from September 30, 2019). Note that in recent years plans to deploy over 17000 new LEO satellite have been announced. Today, among the most wellknown satellite connectivity systems are the International Maritime Satellite Organization (Inmarsat) and Iridium. Inmarsat satellite constellation comprises 13 GEO satellites and offers wireless connectivity for maritime, aviation, enterprise and government with telephone, data services, safety, and missioncritical applications.

The Iridium satellite constellation comprises 66 LEO satellites covering the entire Earth. The satellites are uniformly distributed within six circular orbital planes, having an altitude 
of $780 \mathrm{~km}$ and moving with an orbital velocity of about $27000 \mathrm{kmph}$. A specific location on the surface is served by a satellite for about nine minutes (as long as the elevation angle $(E)$ stays above 8.2 degrees, often rounded to 10 degrees). Conventionally, the communication between a ground terminal and a satellite is carried in L-band (1-2 GHz), while Kaband $(26.5-40 \mathrm{GHz})$ is used for inter-satellite communication. Comprising 1357 operational LEO satellites, CubeSat is one of the notable satellite systems trends that can become especially beneficial for use cases involving M2M connectivity. The CubeSats denote the type of miniaturized satellite made up of multiples of $10 \mathrm{~cm} \times 10 \mathrm{~cm} \times 10 \mathrm{~cm}$ cubic units (termed "U") following the standard specification. Among the key advantages of the CubeSats are their lower cost and design times compared to the custom satellites and lower cost of launch due to more efficient transporting [4].

\section{MMTC-SATELlite CONVERGENCE AND THE ALTERNATIVE IMPLEMENTATION STRATEGIES}

Despite the diversity of these two technologies, the idea of combining the mMTC and satellites is circulating in the research community. To give an example, in [5], authors conceptualize and analyze the performance of the satellite-based mMTC networks for monitoring a smart-city infrastructure. Specifically, the authors imply the use of terrestrial radio technologies for transferring data to a gateway, which is further wired to a satellite modem providing the backbone connection to an application server. A somewhat different approach has been taken in [6], [7], where a machine terminal reaches a satellite using an mMTC radio access technology directly. The offloading and backhaul of cellular machine traffic to a satellite has also been investigated in [8]. In [9], the authors design optimal topology for a sparse satellite constellation and show that only nine LEO satellites are sufficient to enable global coverage. The study [10] assesses the capabilities of LoRa and discusses the different device configurations for satellite-toearth communication with the motivation to provide seamless connectivity in rural areas. In this section, we dive deeper into the pros and cons and trade-offs associated with the two approaches, which are schematically depicted in Fig. 2.

\section{A. Indirect: mMTC Device-Gateway-Satellite (D-G-S)}

This approach implies forming a hybrid mMTC-satellite network that composes a terrestrial network for data acquisition and a satellite backhaul link for transferring the data (or a part of it) further. The main advantage is that this approach relies on well-established and proven technologies and products. Notably, the small data from multiple machines can be aggregated and pre-processed before being streamed to a satellite. Two other arguments pro this approach are (i) the fact that the distance between a machine and the local gateway may be made much smaller than that between a machine and a satellite, thus enabling use of robust and less energy-consuming modulation-coding schemes, and (ii) unlike an LEO satellite, a gateway may be placed statically thus having a more predictable radio channel. The downside of

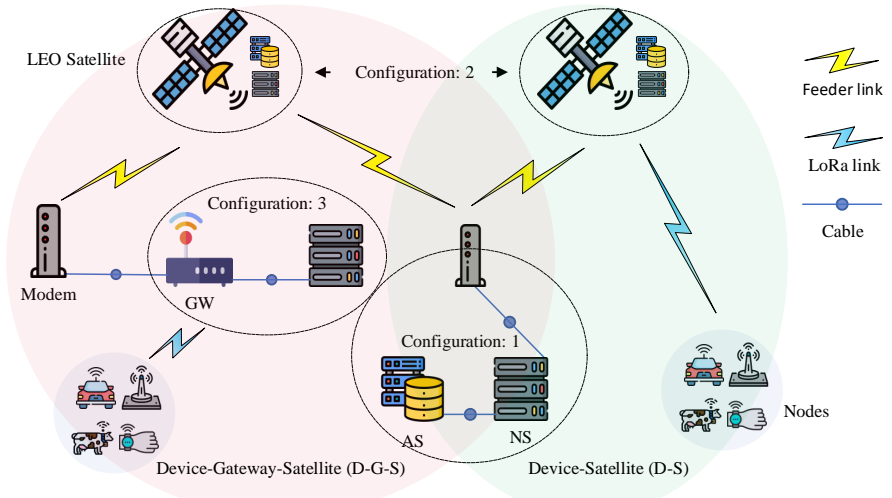

Fig. 2. Direct and indirect mMTC-satellite topologies and network architectures with different possible configuration options for network management functionalities i.e. Network Server (NS) and Application Server (AS).

this approach is the need to create and support a terrestrial infrastructure, which may inquire substantial costs. This may even be impossible if an application is deployed robustly or implying a highly dynamic environment (e.g., sensors floating in the sea). Another potential challenge is the gateway's energy consumption, which may significantly exceed that of the machines. Finally, a gateway may become a potential bottleneck for the whole system's reliability and security, should it get out of order or become compromised.

One of the key open questions relevant to this approach is distributing the network-management functionalities within the hybrid network. To give a practical example, many LPWANgrade technologies imply star-of-stars topology composed of gateways (in LoRaWAN/Sigfox, eNB - in C-IoT) connected to some management entity (LoRaWAN network server, Sigfox cloud or MME for C-IoT) through an IP-based link. Placing this management entity before the satellite may allow reducing the overall traffic through a satellite backbone while positioning it after (or even on) a satellite allows reducing the complexity of the gateway devices. Notably, in the latter case, the latency, throughput, and reliability constraints coming from satellite links need to be considered when configuring the LPWAN network. This aspect also has to be considered when selecting the height of the satellite's orbit. Compared to the two other classes, LEO satellites enjoy the lowest propagation losses and delays. Still, they challenge the system with high orbital velocity, bringing high Doppler and rapid channel attenuation fluctuation. Notably, the per-kilogram costs for raising a load to LEO is typically 5-10 times lower than to GEO, which might become another critical argument for going with LEO satellites

\section{B. Direct: mMTC Device-Satellite (D-S)}

This alternative implementation strategy implies a direct wireless connection between an mMTC device and a satellite. Removal of a mediator (i.e., a gateway) simplifies the network topology and makes the ground component more homogeneous. This also removes a potential bottleneck on the one hand and allows to support more robust deployments and dy- 
namic environments on the other hand. Note, that the sub- $\mathrm{GHz}$ frequency bands used by conventional mMTC technologies are close to the satellite L-band and do not undergo strong attenuation at atmospheric gases, fog, clouds and rain droplets. The absence of the handovers and packet reception by multiple gateways and minimal signalling make LoRaWAN and Sigfox especially promising for such an integration.

However, there are several downsides and challenges associated with this approach. First is the scalability issue. Instead of a limited number of links from the gateways, a radio receiver of a satellite will have to handle myriads of small-data transfers from individual nodes. Second is the need to couple with satellite velocity (and the associated Doppler shift; especially relevant for LEO satellites) and hundreds to thousands-kilometres connectivity distances. This has a twofold effect: (i) the increase of the energy consumption (due to increased transmit power and, potentially, use of repetitions or higher spreading factors), and (ii) the increase of the on-air travel time which may require revising some of the protocol assumptions (e.g., resource scheduling). Notably, the constant change of the distance between a machine and the satellite may complicate modulation-coding scheme adaptation, which will have negative consequences for energy consumption.

The implementation of this approach introduces some exciting design challenges. First, this is the selection (and, importantly, adaptation) of the radio access technology to enable device-satellite communication. Second, one will likely have to address the non-uniform radio spectrum worldwide (especially, if the sub-GHz frequencies often utilized by mMTC today will be employed). This may inquire support and/or switching between the different frequency bands and use of, e.g., beamforming. Third, the distribution of the functionalities within a satellite constellation and between it and the ground element needs to be carefully planned.

\section{LoRaWAN over Satellite: Proof of Concept}

\section{A. Motivation, System Model and Simulation Setup}

In the previous section, we have sketched the two alternative implementation approaches for bringing together the mMTC and satellites. This section focuses on the most flexible and challenging of the two, implying direct connectivity between a satellite and a machine-device. To obtain more in-depth insight into the feasibility, challenges and trade-offs associated with this approach, we narrow down our discussion to only one LPWAN-grade technology, namely LoRaWAN. However, we consider that the obtained insights might also be extrapolated and applied to the other MTC technologies on the one hand and, potentially, other use cases. The major reasons for selecting LoRaWAN are its wide commercial availability, high flexibility, Doppler and interference robustness of the LoRa physical layer, and the decently simple protocol. Notably, the earlier studies have already shown the possibility of using LoRa-modulated signals for long-range (e.g., $832 \mathrm{~km}$ using $25 \mathrm{~mW}$ transmission power [11]) data transfers. The initial empirical trials of LEO satellite-based LoRaWAN gateways carried by, e.g., Lacuna Space, also provided promising results.
TABLE I

System Model's Assumptions and Monte Carlo Simulation PARAMETERS.

\begin{tabular}{|c|c|}
\hline Parameters & Values \\
\hline Carrier frequency $\left(f_{C}\right)$ & $868 \mathrm{MHz}$ \\
\hline Bandwidth $(B)$ & $125 \mathrm{kHz}$ \\
\hline Channels & 8 \\
\hline Transmit power $\left(P_{t x}\right)$ & $14 \mathrm{dBm}$ \\
\hline Device antenna gain $\left(G_{t}\right)$ & $2.15 \mathrm{dBi}$ \\
\hline Gateway antenna gain $\left(G_{r}\right)$ & $22.6 \mathrm{dBi}$ \\
\hline Noise power $(\mathcal{N})$ & $-117 \mathrm{dBm}$ \\
\hline Pathloss exponent $(\eta)$ & 2 \\
\hline Nodes in a satellite coverage $(\bar{N})$ & $100 \mathrm{k}, 250 \mathrm{k}, 500 \mathrm{k}$ \\
\hline Spreading factor $(\mathrm{SF})$ & $7,10,12$ \\
\hline MAC payload $\left(P_{L}\right)$ & 9 bytes \\
\hline Report time $(T)$ & $900 \mathrm{~s}$ \\
\hline Elevation angles $(E)$ & $10^{\circ} \leq E \leq 90^{\circ}$ \\
\hline Rician Factor $(K)$ & $1.24 \leq K \leq 25.11$ \\
\hline Orbital height $(H)$ & $780 \mathrm{~km}$ \\
\hline Earth Radius $(R)$ & $6378 \mathrm{~km}$ \\
\hline Link distance $(d)$ & $R\left[\sqrt{\left(\frac{H+R}{R}\right)^{2}-\cos ^{2}(E)}-\sin (E)\right.$ \\
\hline
\end{tabular}

Specifically, we consider sensor nodes representing the class A LoRaWAN devices equipped with omnidirectional half-wavelength dipole antennas deployed under the open sky on the Earth surface. Next, we envision a LoRaWAN gateway, which is deployed on an LEO satellite, which has the orbit height and characteristics (e.g., the feasible elevation angles) similar to that of the Iridium system. Specifically, each satellite is equipped with directive antennas which cover an area of $15,299,900 \mathrm{~km}^{2}$ with 48 beams, while the area covered by a single beam increases with the increase of the distance to the satellite [12]. Furthermore, we consider that the antenna gain for each beam equals $22.6 \mathrm{dBi}$ and that the signals of the sensors served by the different beams do not interfere with each other. Also, we imply the LoRaWAN network server to be either integrated with the LoRaWAN gateway or deployed in space or on the ground within reach of the gateway. The key parameters of our system model are summarized in Table I and the setup is illustrated in Fig. 3.

Furthermore, we consider that the physical and link-layer procedures match that of the conventional LoRaWAN. The system operates in the EU $868 \mathrm{MHz}$ ISM band (using eight duty-cycle-limited channels with a bandwidth of $125 \mathrm{kHz}$ ). Given the high orbital speed of the satellite (i.e., $7.44 \mathrm{~km} / \mathrm{s}$ ) and the elevation angle ranging $\left(10^{\circ} \leq E \leq 90^{\circ}\right)$ resulting in the distance variation $(2325 \mathrm{~km} \geq d \geq 780 \mathrm{~km})$ between a sensor and a satellite, we consider that no adaptive data rate is used and that all the nodes use a fixed SF (which we vary from one experiment to another) and transmit their signals with $25 \mathrm{~mW}$ (i.e., $14 \mathrm{dBm}$ ) transmit power. A node's (omnidirectional) antenna gain is considered to be $2.15 \mathrm{dBi}$. The Doppler shift due to the satellite movement does not exceed $0.003 \%$, which corresponds for $868 \mathrm{MHz}$ carrier to about 26 $\mathrm{kHz}$. Given the default channel separation of $200 \mathrm{kHz}$ and a channel bandwidth of $125 \mathrm{kHz}$ implied by the LoRaWAN 


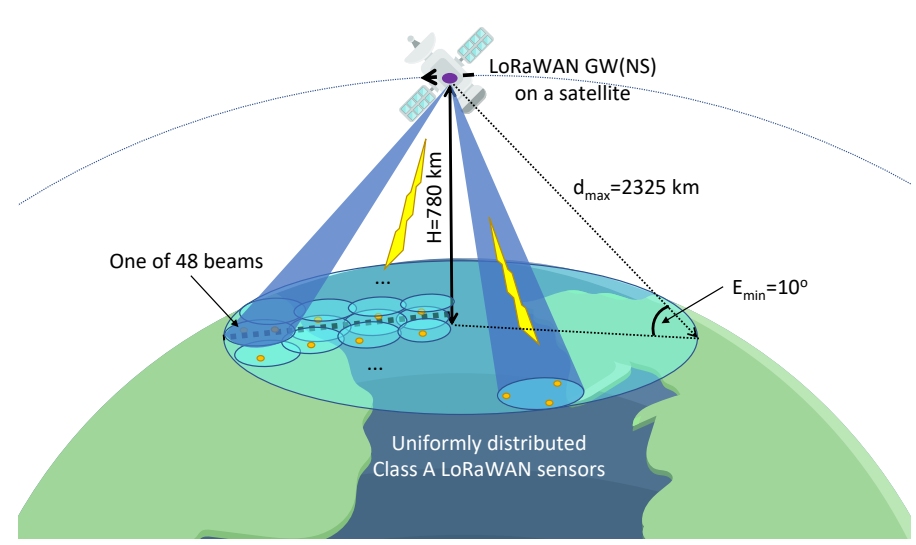

Fig. 3. Simulated scenario and key notations.

specification, this shift should be possible to tolerate with no additional modifications. Importantly, we account also for the time-varying characteristics of the transmission environment due to satellite mobility by considering the dynamic Rician Factor $(K)$ likewise in [13].

Note that under these implications, a one-way delay for radio signal propagation between a sensor and a satellite ranges from $2.6 \mathrm{~ms}$ to $8 \mathrm{~ms}$. For the downlink communication, this delay does not require a change of the first and second LoRaWAN receive windows (RX1 and RX2) delays ( 1 and $2 \mathrm{~s}$ by default, respectively); however, these delays (and the potential for radio channel degradation especially when a satellite moves away from a sensor) may require (i) increasing the data rate offset for $\mathrm{RX} 1$, (ii) using high $\mathrm{SF}$ values for $\mathrm{RX} 2$, and (iii) increasing the duration for channel scan (currently typically equals to five radio-symbol duration) to ensure reliable detection of a downlink preamble despite these delays. Note also that since we imply for our model use of Activation by Personalization (ABP) and no adaptive data rate (ADR), there will be no signalling overhead.

To obtain a more in-depth insight into the feasibility and potential trade-offs associated with this approach, we have carried out studies using the specially developed MATLABbased Monte Carlo simulator. The implied propagation model takes into account the path loss attenuation (based on the Friis model) as well as the Rician fading (whose line of sight component affects the elevation angle). We consider that the sensors are uniformly distributed in the satellite coverage area and transmit their packets randomly with an average period of 15 minutes. The probability of a successful packet reception by a LoRaWAN gateway denoted as success probability $\left(P_{S}\right)$, is composed of the two components. The former one, denoted $P_{S N R}$, represents the probability of signal-to-noise ratio (SNR) of a received by the gateway radio packet exceeding the SF-specific LoRa demodulation threshold $\left(D_{S N R}\right)$ allowing correct packet demodulation. The latter one, expressed $P_{S I R}$, accounts for the interference due to the transmission of the other LoRaWAN nodes. Note that in our simulations, we account for the presence of the capture effect, i.e., the gateway

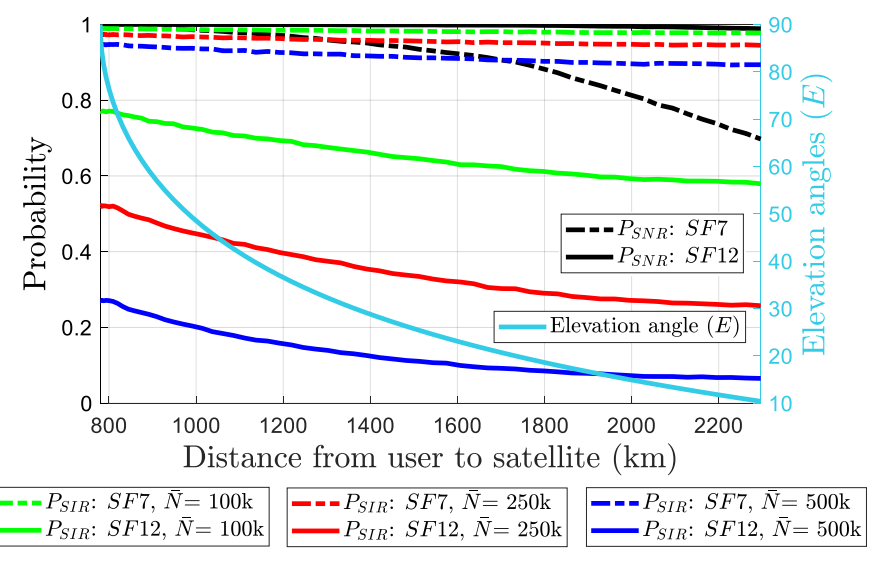

Fig. 4. $P_{S N R}$ - the probability of signal-to-noise ratio (SNR) at gateway exceeding SF-specific LoRa demodulation threshold, and $P_{S I R}$ - probability of mitigating the co-SF interference as a function of distance between a sensor and the satellite for different SF configurations and node densities. The right $\mathrm{y}$-axis illustrates the elevation angle as a function of distance to a satellite.
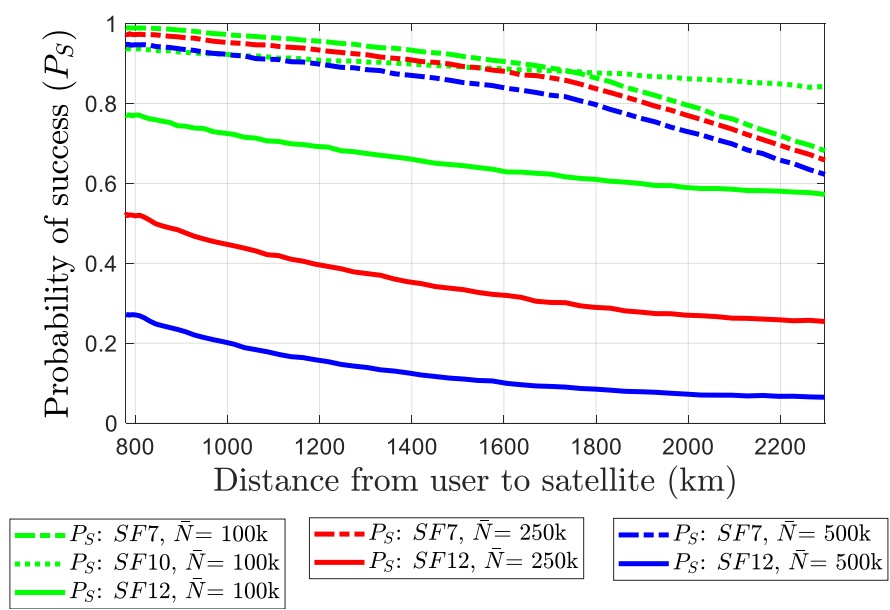

Fig. 5. The joint success probability $\left(P_{S}\right)$ as a function of sensor's distance to the satellite for different SF configurations and node densities. SF10 illustrates the performance of a SF between the minimum and the maximum ones.

successfully decodes a packet under co-SF interference, which is at least $6 \mathrm{~dB}$ weaker [14]. Also, for the presented results, we focus specifically on the uplink transmissions, implying no downlink. Therefore, uplink-downlink interference or the potential effect of the half-duplex operation of a LoRaWAN gateway are not relevant.

\section{B. Selected Numerical Results}

With our numerical results, we focused on investigating (i) the feasibility and (ii) the scalability aspects of implementing LoRaWAN with satellite gateways. The selected results are depicted in Figs. 4 and 5. The former of the two pictures shows the two components (i.e., $P_{S N R}$ and $P_{S I R}$ ) of the success probability independently for the different number of sensors, configured with the minimum and the maximum SF (i.e., SF7 and SF12). Using the second set of axes, for the sake of tractability, we also account for the influence of satellite mobility that rapidly changes the channel characteristics, ele- 
vation angle, and distance to the satellite. Figure 5 reveals the success probability $\left(P_{S}\right)$ as a function of the distance towards the satellite.

The $P_{S N R}$ curves on Figure 4 reveal that even when operating with SF7, a sensor can reach the satellite with a probability exceeding $90 \%$ when the distance to it is below $1700 \mathrm{~km}$. However, this probability drops to less than 0.69 at the maximum distance considered (i.e., $2325 \mathrm{~km}$, which corresponds to $10^{\circ}$ elevation angle and worst link budget case scenario). With SF12, the $P_{S N R}$ stands steady at $100 \%$ until the satellite-sensor distance reaches almost $2100 \mathrm{~km}$, and after that, it declines to 0.98 when the distance touches the coverage border $2325 \mathrm{~km}$.

The $P_{S I R}$ is affected by the two trends. First, due to the increase of an area served by a beam increases the average number of nodes under the uniform node distribution implication, causing slow decrease of $P_{S I R}$ with the distance increase between a satellite and a sensor. Specifically, for 500000 nodes (denoted 500k in the chart) for SF7, with the increase of the distance from the minimum to the maximum, the $P_{S I R}$ drops from 0.95 to about 0.9 , while for SF12, the decline is from 0.27 to 0.08 . Second, owing to the rapid increase of the on-air time (1.5 to two-fold with each next SF), with the increase of the SF, the $P_{S I R}$ decreases for a specific value of the distance between a sensor and a satellite. For example, for 100000 sensors at $1000 \mathrm{~km}$ distance, the $P_{S I R}$ for SF7 is about 0.97 and drops below 0.72 for SF12. The collision probability for 100000 nodes averaged over the whole range of distances was $0.0183,0.1043$, and 0.3530 for SF7, SF10, and SF12, respectively. In $9.53 \%, 9.09 \%$, and $7.48 \%$ of the cases, respectively, a colliding packet has been recovered due to the capture effect.

Both these trends merge in overall success probability $\left(P_{S}\right)$, which is depicted for SF7, SF10 and SF12 and different node densities in Figure 5. The results clearly show the optimal SF's existence depending on the distance between a satellite and a sensor, which is also affected by the network density. As can be seen, in the case of 100000 active sensors, SF7 allows achieving the best performance for sensors located within $1700 \mathrm{~km}$ from the satellite, while SF10 works better for distances exceeding $1700 \mathrm{~km}$.

\section{Open Challenges and Research DiRections}

The presented in the previous section results demonstrate the feasibility of ground to LEO satellite connectivity using LoRa and reveal that selection of configurations (e.g., the SF) maximizing the packet delivery rate depends both on the distance and the number of the sensors served. Note that the presented results, architectures and concepts are also valid and may be generalized for other use-case scenarios involving, e.g., unmanned aerial vehicles (UAVs), high altitude balloons and uncontrolled flying objects. However, further studies are required to validate and confirm these results, as well as to address the relevant challenges arising when merging mMTC and satellites. Below we highlight just some of them:
- Sensor distribution. Likewise in many of the mMTC studies, we implied uniform distribution of the sensor devices and periodic traffic model. This assumption may be valid for terrestrial networks, but does it also hold for a ground-to-satellite scenario? What are the key applications and their requirements? To which extent will, e.g., sensor clustering (consider, e.g., sensors deployed on a dam or an offshore oil rig) and their spatial distribution change the results?

- Terrestrial-satellite cooperation and resource management. In our study, we implied no interference from terrestrial LPWANs. But how well does this assumption hold? Should the terrestrial and satellite-based networks use the same resources (e.g., frequency bands)? Should a handover between these two be implemented? Can some of the solutions developed for cognitive networks or beamforming improve the performance?

- Modulation coding schemes (MCS) and transmit power control. Our results illustrated an interplay between communication range and collision rate for the different SF allocation. However, how (and whether) should the MCS parameters be allocated? How should the high dynamism but the periodic pattern of satellite mobility be considered for media access and scheduling? Is there a need for a scheduling mechanism or a mechanism (e.g., a wake-up radio-based) allowing detection of a satellite by an mMTC device? Given the limitations of a satellite, should sensors take more initiative and freedom to adjust the parameters independently? Another relevant question is: are all current MCS needed, and whether we need the new ones?

- Beamforming. In this study, we considered the satellite beam footprint similar to that of Iridium satellites. However, is this beamforming optimal for MTC case? Should the beam footprint remain static or change dynamically? What are the technical limitations imposed, e.g., by the CubeSat form-factor?

- Network structuring and management. Unlike the terrestrial networks, the amount of resources (energy, processing power, backbone throughput, and even the heat budget) available to a satellite is limited. Given this, how should the functionalities (especially for network management) be distributed within the satellite constellation and between the sky and the ground? Will an edge server implemented on a satellite/within satellite constellation for mMTC data processing be more efficient than transmitting the data to an application server onground? Should the distribution be done dynamically, accounting, e.g., LEO climate conditions (e.g., the satellite's temperature, changing due to the sun heat)?

- Security and reliability. The hundred-kilometre-order links may make these systems more susceptible for the man-in-the-middle (using, e.g., drones or balloons) attacks, while limited resources of a satellite (e.g., downlink) may require rethinking the current procedures for network connection and security credentials provisioning. 
- Legislative and business aspects. Which frequency regulations should be used, and who should control this? How should the legislation non-uniformity around the globe be addressed from legal and technical points of view? Which business model(s) make sense?

\section{CONCLUSIONS}

This article advocates the possibility of convergent mMTCsatellite systems to enable the mMTC applications operating in remote areas. On this way, we discussed the two alternative implementation strategies and provided a more in-depth insight into one of them, implying direct connectivity between the sensors located on-ground and the LEO satellites over LPWAN (specifically LoRaWAN) technology. Our results demonstrate the potential feasibility for a sensor to reach a satellite even when utilizing the configurations typical for today's terrestrial LPWANs and reveal some interplay between the network settings and performance metrics. We hope that these results can motivate further studies to take a deeper and more systematic look at such systems and their operation. In such a case, this study opens research challenges and questions and may serve as further guidance.

\section{ACKNOWLEDGMENT}

This research has been financially supported by Academy of Finland, 6Genesis Flagship (Grant no318927), and EE-IoT (no319008).

\section{REFERENCES}

[1] N. H. Mahmood et al., "White Paper on Critical and Massive Machine Type Communication Towards 6G," 2020.

[2] M. Stusek, D. Moltchanov, P. Masek, K. Mikhaylov, O. Zeman, M. Roubicek, Y. Koucheryavy, and J. Hosek, "Accuracy Assessment and Cross-Validation of LPWAN Propagation Models in Urban Scenarios," IEEE Access, vol. 8, pp. $154625-154$ 636, 2020.

[3] M. A. Ullah, J. Iqbal, A. Hoeller, R. Souza, and H. Alves, "K-Means Spreading Factor Allocation for Large-Scale LoRa Networks," Sensors (Basel, Switzerland), vol. 19, 2019.

[4] N. Saeed, A. Elzanaty, H. Almorad, H. Dahrouj, T. Y. Al-Naffouri, and M. S. Alouini, "CubeSat Communications: Recent Advances and Future Challenges," IEEE Communications Surveys Tutorials, vol. 22, no. 3, pp. $1839-1862,2020$

[5] R. Giuliano, F. Mazzenga, and A. Vizzarri, "Satellite-Based Capillary 5G-mMTC Networks for Environmental Applications," IEEE Aerospace and Electronic Systems Magazine, vol. 34, no. 10, pp. 40-48, 2019.

[6] A. A. Doroshkin, A. M. Zadorozhny, O. N. Kus, V. Y. Prokopyev, and Y. M. Prokopyev, "Experimental Study of LoRa Modulation Immunity to Doppler Effect in CubeSat Radio Communications," IEEE Access, vol. 7, pp. 75 721-75 731, 2019.

[7] J. A. Fraire, S. Céspedes, and N. Accettura, "Direct-To-Satellite IoT A Survey of the State of the Art and Future Research Perspectives," in Ad-Hoc, Mobile, and Wireless Networks, M. R. Palattella, S. Scanzio, and S. Coleri Ergen, Eds. Cham: Springer International Publishing, 2019, pp. 241-258.

[8] B. Soret, I. Leyva-Mayorga, S. Cioni, and P. Popovski. (2020, Nov.) 5G satellite networks for IoT: offloading and backhauling. [Online]. Available: https://arxiv.org/abs/2011.05202

[9] J. A. Fraire, S. Henn, F. Dovis, R. Garello, and G. Taricco, "Sparse Satellite Constellation Design for LoRa-based Direct-to-Satellite Internet of Things," in Proc. Global Commun. Conf., 2020, pp. 1-6.

[10] L. Fernandez, J. A. Ruiz-De-Azua, A. Calveras, and A. Camps, "Assessing LoRa for Satellite-to-Earth Communications Considering the Impact of Ionospheric Scintillation," IEEE Access, vol. 8, pp. 165 570-165 582, 2020.
[11] The Things Network. (2021, Mar.) LoRa World Record Broken: $832 \mathrm{~km} / 517 \mathrm{mi}$ using $25 \mathrm{~mW}$. [Online]. Available: https://www.thethingsnetwork.org/article/ lorawan-world-record-broken-twice-in-single-experiment-1

[12] ETSI TC-SES, "ETR 093: Satellite Earth Stations (SES); Possible European standardisation of certain aspects of Satellite Personal Communications Networks (S-PCN) Phase 1 report," 1993.

[13] Junghwan Kim, Cheng-Ying Yang, and J. S. Jang, "Performance analysis of low-Earth-orbit (LEO) mobile-Satellite system using moment-based approximation of degradation factors," IEEE Transactions on Vehicular Technology, vol. 55, no. 3, pp. 876-886, 2006.

[14] A. Hoeller, R. D. Souza, O. L. Alcaraz López, H. Alves, M. de Noronha Neto, and G. Brante, "Analysis and Performance Optimization of LoRa Networks With Time and Antenna Diversity," IEEE Access, vol. 6, pp. $32820-32829,2018$.

\section{BIOGRAPHIES}

Muhammad Asad Ullah received his BS (Electrical Engineering) degree from National University of Computer and Emerging Sciences, Pakistan in 2017. Since 2018, he is with the Centre for Wireless Communications, University of Oulu, where he completed his master's studies in Wireless Communications Engineering, and currently pursuing Ph.D. in Communications Engineering. His technical interests include mMTC, LoRaWAN, and Non-terrestrial-Networks.

Konstantin Mikhaylov is an Assistant Professor for Convergent IoT Communications for Vertical Systems with the Centre for Wireless Communications at the University of Oulu. The major focus of his research is on the radio access and beyond-access technologies for massive and dependable IoT, and the matters related to the design and use of the IoT systems. He has authored or coauthored about one hundred research works on IoT wireless connectivity, system design, and applications. Dr. Mikhaylov is a Senior Member of IEEE.

Hirley Alves (S'11-M'15) is Assistant Professor and Head of the Machine-type Wireless Communications Group at the 6G Flagship, Centre for Wireless Communications, University of Oulu. He is actively working on massive connectivity and ultra-reliable low latency communications for future wireless networks, $5 \mathrm{G}$ and $6 \mathrm{G}$, full-duplex communications, and physical-layer security. He has received several awards and has been the organizer, chair, TPC and tutorial lecturer for several renowned international conferences. He is the General Chair of the ISWCS'2019 and the General Co-Chair of the 1st 6G Summit, Levi 2019, and ISWCS 2021, Track Chair PIMRC'2021. 